ARTIGO DE REVISÃO

\title{
LISTERIA MONOCYTOGENES: OCORRÊNCIA EM PRODUTOS LÁCTEOS E SUAS IMPLICAÇÕES EM SAÚDE PÚBLICA
}

\author{
G.V. Barancelli², J.V. Silva-Cruz ${ }^{1}$, E. Porto ${ }^{2}$, C.A.F. Oliveira ${ }^{1}$ \\ ${ }^{1}$ Universidade de São Paulo, Faculdade de Zootecnia e Engenharia de Alimentos, Av. Duque de Caxias \\ Norte, 225, CEP 13635-900, Pirassununga, SP, Brasil. E-mail: carlosaf@usp.br
}

RESUMO

\begin{abstract}
Listeria monocytogenes é o agente causador da listeriose, uma grave doença de origem alimentar que causa severas infecções em humanos com altas taxas de mortalidade. O leite e seus derivados estão entre os produtos alimentícios mais frequentemente envolvidos na transmissão de $L$. monocytogenes. A listeriose acomete, sobretudo, indivíduos imunodeprimidos, grávidas, recémnascidos e idosos, o que ressalta o caráter oportunista deste micro-organismo e sua importância para a saúde pública. No presente trabalho, faz-se uma revisão narrativa crítica sobre o risco à saúde humana decorrente da ingestão de leite e derivados contaminados por L. monocytogenes, bem como se discutem os fatores que determinam a contaminação por L. monocytogenes na cadeia de produção e distribuição de leite e derivados. São apresentados e avaliados os dados de ocorrência de L. monocytogenes em leite cru e em produtos lácteos no Brasil, tendo em vista seu potencial de envolvimento em casos de listeriose humana. Adicionalmente, são indicadas as principais áreas de pesquisa e atuação para prevenir a contaminação de L. monocytogenes em produtos lácteos.
\end{abstract}

PALAVRAS-CHAVE: Doenças transmitidas por alimentos, listeriose, derivados de leite.

\begin{abstract}
LISTERIAMONOCYTOGENES:OCCURRENCEINDAIRYPRODUCTSANDITSIMPLICATIONS IN PUBLIC HEALTH. Listeria monocytogenes is the causative agent of listeriosis, a serious foodborne disease that promotes severe human infections with high mortality rates. Milk and byproducts are among the food products most often involved in the transmission of L. monocytogenes. Listeriosis mainly affects immunodepressed individuals, pregnant women, neonates and the elderly, thus emphasizing its opportunistic character and importance to public health. The present article presents a narrative and critical review concerning the risk to human health from the consumption of dairy products contaminated with L. monocytogenes. Also, a discussion is made on the factors that determine the contamination by L. monocytogenes in the production and distribution chain of milk and dairy products. The available data on the occurrence of L. monocytogenes in raw milk and dairy products in Brazil are also presented and evaluated, taking into consideration its potential for involvement in human listeriosis outbreaks. Additionally, this review indicates the main research and work areas needed for the prevention of L. monocytogenes contamination in dairy products.
\end{abstract}

KEY WORDS: Foodborne diseases, listeriosis, dairy products.

\section{INTRODUÇÃO}

O gênero Listeria compreende as seis espécies L. monocytogenes, L. ivanovii, L. seeligeri, L.innocua, L.welshimeri e L. grayi (ROCOURT; BUCHRIESER, 2007) das quais somente L. monocytogenes é considerada consistentemente patogênica para o homem, embora infecções ocasionais por L. innocua, L. seeligeri, $L$ welshimeri e $L$. ivanovii venham sendo relatadas (Chambel et al., 2007). L. monocytogenes é um bastonete Gram-positivo pequeno com extremidades arredondadas e não produz e esporos ou cápsulas. É móvel quando cultivada entre 20 e $25^{\circ} \mathrm{C}$, porém é imóvel ou apresenta fraca motilidade a $37^{\circ} \mathrm{C}$. O seu cultivo em meio semissólido resulta em crescimento com forma característica de "guarda-chuva" a aproximadamente $0,5 \mathrm{~cm}$ abaixo da superfície do meio, devido à natureza microaerófila do organismo (ROCOURT; Buchrieser, 2007). A bactéria é sensível à pasteurização (RYSER; DONNELLY, 2001) e resiste a condições ambientais adversas como baixo $\mathrm{pH}$ e altas concentrações de $\mathrm{NaCl}$ (ROCOURT; BUCHRIESER,

${ }^{2}$ Universidade de São Paulo, Escola Superior de Agricultura “Luiz de Queiroz", Piracicaba, SP, Brasil. 
2007). O solo e vegetais em decomposição são os principais reservatórios da L. monocytogenes, que tem sido isolada de hortaliças, água doce, esgoto e do material fecal de várias espécies de mamíferos, aves e peixes, em geral portadores assintomáticos, que liberam a bactéria nas fezes (RYSER; DonNELly, 2001). Isso explica o fato dessa bactéria ser facilmente encontrada em alimentos de origem animal e vegetal, "in natura" ou processados. O leite e seus derivados estão entre os produtos alimentícios mais frequentemente envolvidos na transmissão de $L$. monocytogenes.

A listeriose resulta, principalmente, da ingestão de alimentos contaminados com L. monocytogenes (MEAD et al., 1999) geralmente processados, armazenados sob refrigeração por longos períodos e consumidos sem aquecimento (McLAUCHLIN, 1996), tal como ocorre com os produtos de laticínios, principalmente queijos. Outros fatores de risco associados aos alimentos envolvidos em surtos de listeriose incluem a ausência de tratamento térmico que promova a destruição de Listeria, a qualidade nutricional de alimentos que favoreçam o crescimento de L. monocytogenes e o consumo por indivíduos imunodeprimidos, mulheres grávidas e idosos (Food..., 2005). A listeriose apresenta um período de incubação longo, que varia de 3 a 70 dias (CENTRO..., 2003), o que dificulta identificar o agente causador e rastrear a origem da contaminação do alimento.

Psicrotrófica, L. monocytogenes é capaz de multiplicar-se sob refrigeração, o que torna um desafio o seu controle na cadeia de produção de alimentos. A expansão do uso da cadeia de frio na estocagem de alimentos, na indústria, no comércio e nas residências, o uso de alimentos "prontos para consumo", minimamente processados refrigerados, representam nichos quefavorecema L.monocytogenes (KOZAK et al., 1996).

A diferença entre alimentos de alto ou baixo risco para a listeriose é a habilidade do micro-organismo se multiplicar eatingir altos números. Emalimentos que oferecem condições para a multiplicação de L. monocytogenes, uma população inicial baixa poderá atingir contagens consideráveis durante o armazenamento sob refrigeração prolongado (ILSI, 2005). Alimentos assim devem ser mantidos em temperaturas de $4,4^{\circ}$ $\mathrm{C}$ ou inferiores, para retardar o crescimento da bactéria, em caso de contaminação (UNITED, 2003), que é acentuado em temperaturas de abuso (ILSI, 2005).

Numa avaliação quantitativa de risco realizada nos EUA, foi estimado que produtos lácteos apresentam alto risco relativo para listeriose humana (UNITED, 2003), sendo incriminados em 9 dos 26 surtos relatados. Outra avaliação estimou o risco de ocorrência de listeriose humana a partir da ingestão de quatro alimentos prontos para o consumo: leite pasteurizado, sorvete, peixe defumado e carnes fer- mentadas. O leite pasteurizado foi apontado como o de maior risco de causar listeriose na população, sendo a estimativa de ocorrência de 9,1 casos/10 milhões de pessoas/ano. Apesar de dados epidemiológicos evidenciarem baixa ocorrência da bactéria em leite pasteurizado, o produto oferece condições para a multiplicação do micro-organismo durante o armazenamento e é amplamente consumido pela população. Já o sorvete, além da baixa ocorrência neste produto, não oferece condições de crescimento para a bactéria (FAO/WHO, 2004).

No Brasil, a listeriose humana é subdiagnosticadae subnotificada (SILva et al., 2007), não havendo registros de casos transmitidos por alimentos (DESTRO, 2006), embora L.monocytogenes esteja comprovadamentepresente em diversos produtos, sobretudo em derivados lácteos(CARVAlHOetal.,2007; BRITOetal.,2008; ABRAãoet al., 2008; MARTins et al., 2009). É provável que a maioria doscasos humanos esporádicos tenha oalimento como veículo de transmissão da L. monocytogenes (HOFER et al., 2006) o que reforça a necessidade de identificar as fontes de infecção e os possíveis alimentos envolvidos, principalmente o leite e derivados.

No presente trabalho, faz-se uma revisão narrativa crítica sobre o risco à saúde humana decorrente da ingestão de produtos lácteos contaminados por $L$. monocytogenes e uma análise dos dados disponíveis sobre a ocorrência deste agente em produtos lácteos no Brasil, além dos fatores que favorecem a contaminação por L. monocytogenes na cadeia de produção e distribuição de leite e derivados, da granja até o consumidor.

\section{Listeria monocytogenes como agente de infecção alimentar}

Há dois tipos de listeriose associados com $L$. monocytogenes: não invasiva e invasiva. Ao contrário de outras infecções alimentares, a listeriose invasiva não causa sintomas gastrointestinais em humanos. Depois de ingeridas, as células colonizam o intestino, sãofagocitadas por macrófagos, onde se multiplicam e espalham-se pelo organismo (KATHARIOU, 2002), ficando protegidas dos mecanismos de defesa do hospedeiro, o que limita a terapia com drogas (HoF, 2003a). As manifestações clínicas da listeriose em adultos são decorrentes, principalmente, de infecções no sistema nervoso central (SNC) como meningite e encefalite, além de outras infecções como endocardite, peritonite, pneumonia e ostiomielite (FARBER; Peterkin, 1991).

O grupo de pessoas de alto risco de susceptibilidade ao micro-organismo inclui neonatos, mulheres grávidas, idosos e indivíduos imunodeprimidos (SLUTSKER; SCHUCHAT, 1999). A maioria dos indivíduos imunocompetentes supera o ataque inicial e elimina a L. monocytogenes pelas fezes, entretanto, em indi- 
víduos do grupo de risco, ela causa a forma invasiva da doença. Em gestantes, a bactéria pode provocar abortamento, nascimento prematuro, morte fetal, meningite ou septicemia neonatal (ROCOURT et al., 2003). Trata-se de um patógeno oportunista (FAO/ WHO, 2004), embora registram-secasos emindivíduos imunocompetentes (HOFER et al. 1998).

A maioria dos casos de listeriose humana ocorre em idosos, gestantes e indivíduos imunocomprometidos, sendo que aproximadamente $1 / 3$ dos casos ocorre em gestantes (ILSI, 2005). Dentro da população susceptível, é possível identificar subgrupos mais prováveis do que outros de adoecer gravemente e morrer devido à listeriose. Assim, foi estimado que indivíduos transplantados, portadores de câncer no sangue e com AIDS (Acquired Immunodeficiency Syndrome) apresentam 2.584, 1.364 e 865 vezes maior susceptibilidade relativa para a doença, respectivamente, do que indivíduos com menos de 65 anos sem comprometimento imunológico (FAO/WHO, 2004; ILSI, 2005). Atualmente, existem fatores que colaboram para a expansão da população altamente susceptível à L. monocytogenes, entre eles a epidemia de AIDS, o uso de medicamentos imunossupressivos em transplantados, as terapias para o câncer e o aumento da populaçãoidosa. A relevância da L. monocytogenes em saúde pública se deve à gravidade da patogenia e à alta letalidade (30\%) da listeriose (ROCOURT et al.,2003).

Existem relatos de surtos de gastroenterite febril causados pela bactéria (DALTON et al., 1997; AuRELI et al., 2000; CARRIQUE-MAs et al, 2003), uma forma mais branda da listeriose em humanos, não invasiva, com sintomas não específicose, por isso, subdiagnosticada (SWAMINATHAN; GERNER-SMIDT, 2007) e parece não afetar grupos populacionais específicos (DESTRO, 2006). Ressalta-se que existem outras vias de infecção por $L$. monocytogenes, além da oral, como a via respiratória, através de aerossóis (MARTH, 1988). RADOSTITS et al. (1994) relataram que pessoas do meio rural apresentavam maior possibilidade de contaminação por $L$. monocytogenes, devido ao contato com a bactéria em situações específicas, como o manejo de animais de produção infectados, com casos de dermatite na pele de veterinários após o auxílio a partos distócicos, além decasos de conjuntivite. Entretanto, atualmente sabeseque populações do meiourbano sãofrequentemente expostas ao micro-organismo através de alimentos contaminados, eas indústrias de alimentos podem ser pontos importantes da contaminação, especialmente dos alimentos prontos para o consumo.

Há13 grupos sorológicos de L. monocytogenes (RYSER, MARTH, 1991), sendo que a maioria dos casos de listeriose humanaéassociada aos sorotipos $1 / 2 \mathrm{a}, 1 / 2 \mathrm{~b}$ e4b(SWAMINATHAN; GERNER-SMIDT, 2007). Opredomínio desses poucos sorotipos relacionados à doença humana pode explicar o baixo número de casos de listeriose, em situações deexposição frequente a alimentos contaminados com L. monocytogenes (ToMPKIN, 2002). Estudos de subtipagem mostram queL. monocytogenes podeserseparada em três grupos genéticos (linhagens I, II e III). A Linhagem I inclui os sorotipos 1/2b, 3b, $4 \mathrm{~b}, 4 \mathrm{~d}$ e $4 \mathrm{e}$ e a Linhagem II corresponde aos sorotipos 1/2a, 3a, 1/2ce 3c (ILSI, 2005). Históricos clínicos sugerem que há diferença no potencial patogênico entre as 3 Linhagens, uma vez que a Linhagem I contém a maioria dosisolados relacionados à listeriose humana (JeFFERs et al., 2001), enquanto cepas da Linhagem II são mais frequentemente isoladas de alimentos (ILSI, 2005). Esses dados sugerem que possa existir uma linhagem de L. monocytogenes (Linhagem I) com maior probabilidade de causar doença em humanos, quando presente nos alimentos (ILSI, 2005). No entanto, preventivamente, é necessário considerar todas as cepas de L.monocytogenes como potencialmente patogênicas (TOMPKIN, 2002).

A dose infectante (DI) para humanos não está estabelecida, devido à impossibilidade de se realizar pesquisas com voluntários saudáveis, à variação da suscetibilidade e condição imunitária do hospedeiro e à variabilidade na virulência do patógeno. Outra dificuldade de se estabelecer a DI é que os dados coletados de surtos de listeriose podem não representar a real contaminação do alimento incriminado. Devido ao tempo entre a exposição e o início dos sintomas, pode haver multiplicação, morte ou injúria da bactéria no alimento, ou mesmo não haver mais o alimento disponível. Em pesquisa com camundongos, SCHLECH (1988) relatou uma DI oral < $10^{2}$ células, mas GOLNAZARIAN et al. (1989) estimaram DI entre $10^{3}$ e $10^{9}$ células e StEPHENS et al. (1991) o valor de $10^{10}$ células. Dados coletados em surtos de listeriose sugerem que os alimentos incriminados continham contagens elevadas de L. monocytogenes, cerca de $10^{6}$ $\mathrm{UFC} / \mathrm{g}$ (UNITED, 2003), o que realça a necessidade de minimizar a exposição humana a altas populações da bactéria. Dados mais recentes (FAO/WHO, 2004) sugerem que contagens menores do que $10^{2} \mathrm{UFC} / \mathrm{g}$ em alimentos não são infectantes, mas não excluem essa possibilidade. Assume-se que menos do que 1.000 células possam causar a doença em populações susceptíveis (UnITED, 2007). Como alertaram GolnAZARIAn et al. (1989), na estimativa de risco, deve-se considerar que pessoas saudáveis podem resistir à ingestão de um número de células de $L$. monocytogenes que podecausar doença emindivíduos imunodeprimidos. Apesar da controvérsia sobre os valores de DI para L. monocytogenes, considera-se o critério de "ausência em 25 ou 50 g" adequado como limite de tolerância para o micro-organismo em alimentos (FAO/WHO, 2004). Ressalte-se, no entanto, que contagens inferiores a $10^{2} / \mathrm{g}$ em alimentos sólidos e $<10 / \mathrm{mL}$ em alimentos líquidos podem não ser detectadas por plaqueamento direto nos meios usuais de isolamento (НЕIsICK et al., 1995). 
A alternativa mais eficiente seria a contagem em tubos através da técnica do Numero Mais Provável (NMP) (TRANT; Hitchins, 1996).

No que concerne às listerioses transmitidas por produtos lácteos, há surtos de grande repercussão. Um dos mais antigos descritos ocorreu na Alemanha, na década de 1950, sugerindo associação com leite cru (SeELIger, 1961). Em 1983, em Massachusets, um surto de listeriose associado a leite pasteurizado contaminado acometeu 49 pessoas e causou 14 mortes (FLEMMING, 1985). Este surto e outro ocorrido na Califórnia, em 1985, com 142 casos e 48 mortes, atribuído ao consumo de queijo estilo mexicano contaminado (LinNAN et al., 1988), foram importantes para esclarecer o papel do alimento na disseminação da listeriose. Na década de 1980, ocorreram diversos surtos relacionados a queijos contaminados (HoF, 2003b) e, desde então, há relatos de surtos de listeriose de origem alimentar ligados a várias categorias de alimentos como produtos lácteos, cárneos prontos para consumo e frutos do mar (Lianou; Sofos, 2007). Em 1994, o consumo de leite pasteurizado achocolatado contaminado resultou em surto de gastroenterite nos EUA (DALTON et al., 1997). Em Massachusetts (EUA), em 2007, ocorreu um surto atribuído ao consumo de leite pasteurizado contaminado que acometeu 5 pessoas resultando em 3 mortes (CDC, 2008). Na Finlândia, o consumo de manteiga causou um surto envolvendo 25 pessoas, com 6 mortes (LYYTIKÄINEN et al., 2000). Na Suíça, entre 1983 e 1987, um surto acometeu 122 pessoas, com 34 óbitos, atribuído ao consumo de queijo (RYSER; MARTH, 1991). Outros surtos associados ao consumo dequeijos contaminados ocorreram naFrança (Goulet et al., 1995; JACQUET et al.,1995), Canadá (GAULIN, 2003), Suécia (CARRIQUE-MAS, et al., 2003) Japão (MAKINO et al., 2005), EUA (McDonald et al., 2005) e Suíça (BILle et al., 2006). Surtos recentes de listeriose na Áustria e Alemanha, atribuídos ao consumo de queijo tipo "Quargel", resultaram em 4 mortes (Fretz et al., 2010).

A maioria dos casos de listeriose humana ocorre de forma esporádica, ao invés de surtos alimentares (Rocourt et al., 2003). Em 2007, a rede de vigilância FoodNet registrou o número de casos de listeriose nos EUA ( $n=122$ ) com uma incidência de 0,27 casos / 100.000 habitantes (CENTER..., 2007). No Brasil, até o momento, a notificação de casos de listeriose não é compulsória, o que resulta na falta de dados que mostrem a magnitude do problema.

Atualmente, existem políticas sanitárias relacionadas com a presença de L. monocytogenes em alimentos em muitos países. Os EUA estabeleceram tolerância zero para a bactéria, em alimentos prontos para oconsumo, incluindo produtos lácteos, com base na possibilidade de que a dose mínima infectante possa ser baixa (RYSER; MARTH, 1991). No Canadá, os alimentos são classificados em categorias, de acordo com as condições que oferecem para a multiplicação da bactéria, tempo de vida de prateleira e temperatura dearmazenamento. Deacordo com a categoria, os critérios microbiológicos para L. monocytogenes variam de ausência em $50 \mathrm{~g}$ até $<100 \mathrm{UFC} / \mathrm{g}$ no alimento e, para cada situação, são recomendadas diferentes ações (LAKE et al., 2005). No Brasil, a única categoria de alimentos, com padrão para L. monocytogenes, são queijos de média, alta e muito alta umidade, para os quais a legislação estabelece ausência da bactéria em $25 \mathrm{~g}$, em 5 amostras do produto de um mesmo lote (BRASIL, 1996; AGÊNCIA, 2001).

Listeriose humana e portadores de L. monocytogenes no Brasil

Diversos trabalhos mostram a circulação de $L$. monocytogenes na espécie humana provocando infecções e revelando portadores assintomáticos no Brasil. Apesar da listeriose ser subdiagnosticada e subnotificada no país, há registros de casos de listeriose humana antes mesmo da sua associação com transmissão alimentar, ocorrida na década de 1980 (Hof, 2003b). SuAssuna et al. (1969) isolaram L. monocytogenes de líquor, sendo provavelmente o primeirocaso descrito no antigo Estado da Guanabara. Hofer; Menezes (1969) também isolaram o microorganismo a partir de secreção vaginal de mulheres com histórico de abortos crônicos, havendo duas amostras positivas em 42 casos estudados. ESPER et al. (1978) diagnosticaram 12 casos de meningitecausados por L. monocytogenes, entre 1975e1977. Registros mais recentes de listerioseno Brasil incluem 5 casos de bebês com meningite em São Paulo (LANDGraf et al., 1999); listeriose em pacientes transplantados renais (HOFER et al., 1999); em caso de pneumonia em adolescente acometida de cirrose (DE SÁ et al., 2004) e casos de peritonite bacterina espontânea emidosos portadores de cirrose (Toyoshima et al., 2006). Em Porto Alegre, L. monocytogenes foi detectada em 33,7\% (50/148) das placentas humanas relacionadas a abortos e partos pré-maturos (SCHWAB; EdelWEISS, 2003).

A caracterização sorológica de 71 amostras de $L$. monocytogenes, isoladas de processos patológicos e de portadores humanos no Brasil entre 1969 e 1983, revelou predomínio do sorotipo $4 \mathrm{~b}(50,70 \%$ ) (HOFER et al., 1984). Hofer et al. (2000) avaliaram a distribuição e a frequência de espécies e sorovares em isolados com diagnóstico presuntivo de Listeria $(\mathrm{n}=3.112)$ provenientes de diferentes fontes, colecionados entre 1971 e 1999. Dentre os isolados de humanos, 3 tipos antigênicos de L. monocytogenes predominaram: $4 \mathrm{~b}(45,4 \%), 1 / 2 \mathrm{a}(20,9 \%)$ e $1 / 2 \mathrm{~b}(19,1 \%)$. Hofer et al. (2006) caracterizaram a distribuição de sorovares de L. monocytogenes entre 255 amostras do gênero Listeria isoladas de diferentes materiais clínicos de origem humana de várias regiões do Brasil coletadas 
no período de 1969 a 2000 e observaram que o sorovar $4 \mathrm{~b}$ foi o mais incidente $(60,3 \%)$, seguido por $1 / 2$ $(29 \%)$. Entre 13 cepas de L. monocytogenes isoladas de casos de listeriose humana ocorridos entre 1995 e 2005 no Estado de São Paulo, Lemes-Marques et al. (2007) também encontraram predomínio do sorotipo $4 \mathrm{~b}$ seguido por $1 / 2 \mathrm{a}$. Estes dados corroboram com os achados de Hofer et al. (2000) que indicam o sorotipo $1 / 2$ a como o segundo mais frequentemente isolado de amostras de listeriose em humanos. Ressalte-se que este também é o sorotipo mais frequentemente isolado de produtos lácteos no Brasil (Hofer et al., 2006; BRITO et al., 2008).

\section{Ocorrência de Listeria monocytogenes em produtos lácteos}

O leite é um dos produtos mais frequentemente envolvidos na transmissão de L. monocytogenes pois, além de rico em nutrientes, sua cadeia de produção oferece diversas possibilidades de contaminação, como ordenha, transporte, armazenamento e beneficiamento (BEMRAH et al., 1999).

A silagem contaminada é uma fonte clássica de infecção por L. monocytogenes para animais, que podem adoecer ou tornarem-se portadores assintomáticos, eliminando a bactéria nas fezes e no leite. Vacas com mastite por L. monocytogenes eliminam números altos dessa bactéria no leite (RADOstiTs et al., 1994). Assim, ruminantes podem perpetuar os ciclos de transmissão da L. monocytogenes e altas cargas da bactéria oriundas de ambientes rurais podem representar uma fonte de introdução do patógeno na cadeia de produção de laticínios (IVANEK et al., 2006).

A presença de L. monocytogenes em leite cru é preocupante, pois existe o hábito de consumi-lo diretamente ou utilizá-lo na produção de derivados sem nenhum tratamento térmico prévio. Em leite cru, a ocorrência de L. monocytogenes é variável, dependendo do local, do ponto de coleta na cadeia de produção do leite, da amostragem e do método utilizado para sua detecção. Na Suécia, L. monocytogenes foi isolada em 1\% de 294 amostras de leite cru de tanques de fazendas e em $19,6 \%$ dos silos de recepção de uma indústria (WAAK et al., 2002). Na Espanha, Dominguez-RodRiguez et al. (1985) encontraram $45,3 \%$ de amostras positivas de leite cru, de 95 coletadas. Nos EUA, em leite cru de tanques de fazendas, foram relatadas prevalências de 5,9\% $(\mathrm{n}=948)$ (MuraOKa et al., 2003) e 6,5\% $(\mathrm{n}=861)(\mathrm{VAN}$ Kessel et al., 2004). Massa et al. (1990) não isolaram a bactéria em leite cru $(n=40)$ produzido na Itália, o que pode ser explicado pelo baixo número de amostras analisado. No Brasil, a ocorrência de L. monocytogenes em leite cru varia de 0 a $37,8 \%$, conforme atestam os trabalhos relacionados na Tabela 1.

A maioria das pesquisas com leite cru realizadas no Brasil analisou um número pequeno de amostras. É recomendável que pesquisas com essa bactéria sejam feitas com um universo grande de amostras, para que a amostragem seja representativa. A análise de poucas amostras aumenta a probabilidade da ausência de L. monocytogenes e esse resultado não significa, necessariamente, que o micro-organismo não esteja presente no lote. Por outro lado, uma ou duas amostras positivas podem representar uma alta porcentagem deocorrência. Na comparação de dados de ocorrência, é preciso considerar que, durante os anos 1990, houve considerável aperfeiçoamento dos métodos de isolamento de L. monocytogenes, facilitando sua identificação. Assim, dados anteriores a esse período podem, eventualmente, subestimar a presença de L. monocytogenes nas amostras.

Outros critérios de amostragem podem influenciar diretamente o resultado dos levantamentos sobre a ocorrência de L. monocytogenes. Nero (2005) não isolou L. monocytogenes em 210 amostras de leite cru (Tabela 1). Contudo, o trabalho não utilizou uma amostragem persistente em um único estabelecimento, mas em 210 diferentes fazendas. Por ocorrer em baixos números no ambiente, seu isolamento é difícil e, nesse aspecto, estudos de incidência são melhores indicadores do que estudos de prevalência (Toma et al., 1999). A detecção de L. monocytogenes pode ser difícil devido às baixas contagens da bactéria encontradas em leite cru (normalmente inferiores a $10 \mathrm{UFC} / \mathrm{mL}$ ) eà competição da microbiota bacteriana (Meyer-Broseta et al., 2003).

Tabela 1 - Ocorrência de L. monocytogenes em leite cru no Brasil.

\begin{tabular}{lccc}
\hline Estado & $\begin{array}{c}\mathrm{N}^{\circ} \text { de amostras } \\
\text { analisadas }\end{array}$ & $\begin{array}{c}\text { Amostras } \\
\text { positivas (\%) }\end{array}$ & Referência \\
\hline São Paulo & 20 & 0,0 & DeSTRO et al. (1991) \\
São Paulo & 220 & 9,5 & MourA et al. (1993) \\
São Paulo & 20 & 0,0 & CASAROTTI et al. (1994) \\
Paraíba & 60 & 15,0 & FigUEIREDO (2000) \\
Ceará & 45 & 37,8 & CATÃO; CEBALOS (2001) \\
Bahia & 6 & 16,7 & SiLVA et al. (2003) \\
Minas Gerais, Paraná, Rio Grande do Sul e São Paulo & 210 & 0,0 & NeRO (2005) \\
Minas Gerais e Rio de Janeiro & 42 & 0,0 & ARCURI et al. (2006) \\
São Paulo & 286 & 0,0 & CAMARGO (2010) \\
\hline
\end{tabular}


O binômio tempo-temperatura $\left(75^{\circ} \mathrm{C} / 15 \mathrm{~s}\right)$ da pasteurização do leite é suficiente para a destruição de L. monocytogenes com contagens de $10^{5}$ a $10^{6} / \mathrm{mL}$ (JAY, 2000). Portanto, quando a bactéria é detectada em leite pasteurizado, as causas mais prováveis são pasteurização inadequada ou contaminação pósprocesssamento (RYSER; MARTH, 1991). No Brasil, pesquisas conduzidas na região Sudeste mostram que o leite pasteurizado não é uma fonte comum da bactéria (DESTRO et al., 1991; CASAROTTI et al., 1994; ROCHA, 2004; BRITO et al., 2008), enquanto que pesquisas realizadas no Nordeste relatam ocorrência da bactéria entre 3,3\% ( $n=120)$ (Figueiredo, 2000) e $30 \%$ (n = 30) (CATÃo; Ceballos, 2001) das amostras analisadas.

Em produtos lácteos fermentados, a viabilidade de Listeria depende das interações entre os fatores que afetam sua sobrevivência como atividade de água; quantidade, tipo e atividade das culturas láticas; $\mathrm{pH}$; quantidade de sal e temperatura durante o processamento e o armazenamento. Em iogurte, Listeria pode sobreviver ao processo de fermentação e ser encontrada após 30 dias de fabricação, em pH em torno de 4,0. Em manteiga, foi demonstrada a sobrevivência de L. monocytogenes durante o armazenamento do produto congelado $\left(-18^{\circ} \mathrm{C}\right)$ por 70 dias (RYSER; MARTH, 1991).

Os queijos são os produtos lácteos mais comumente contaminados por L. monocytogenes, principalmente os de alta e média umidade. A presença dessa bactéria nesses queijos é preocupante pois, geralmente, são produtos armazenados por longos períodos sob refrigeração, permitindo o seu crescimento, além do fato de serem consumidos sem aquecimento prévio. Queijos macios maturados por fungos parecem oferecer boas condições para o desenvolvimento da bactéria, possivelmente em função do aumento do $\mathrm{pH}$ durante a maturação. Já queijos duros, como o parmesão, apresentam $\mathrm{pH}$ ácido e baixa atividade de água e não permitem o crescimento de L. monocytogenes (RYSER; MARTH, 1991). Numa avaliação de risco de transmissão de L. monocytogenes, por alimentos prontos para o consumo, queijos duros foram classificados como os de menor risco (United, 2003). No Brasil, relata-se ampla variação de ocorrência (zero a $41 \%$ ) de L. monocytogenes em queijos (Tabela 2).

Tabela 2. Ocorrência de L. monocytogenes em queijos no Brasil.

\begin{tabular}{|c|c|c|c|c|}
\hline \multirow[t]{2}{*}{ Cidade (Estado) } & \multirow[t]{2}{*}{ Tipo de queijo } & \multicolumn{2}{|c|}{ L. monocytogenes } & \multirow[t]{2}{*}{ Referência } \\
\hline & & $\mathrm{N}^{*}$ & $\mathrm{n}(\%)$ & \\
\hline Campinas, SP & Minas frescal & 20 & $2(10,0)$ & DESTRO et al. (1991) \\
\hline Piracicaba, SP & Minas frescal & 20 & $0(0,0)$ & CASAROTTI et al. (1994) \\
\hline São Paulo, SP & Minas frescal & 30 & $2(6,7)$ & FURLANETO et al. (1996) \\
\hline \multirow[t]{3}{*}{ R. de Janeiro, RJ } & Minas frescal $^{\mathrm{X}}$ & 17 & $7(41,2)$ & SiLva et al. (1998) \\
\hline & Minas frescal e ricota ${ }^{Y}$ & 33 & $1(3,0)$ & \\
\hline & Brie,Gorgonzola, Roquefort & 53 & $3(5,7)$ & \\
\hline São Paulo, SP & Minas frescal & 20 & $5(25,0)$ & VIEIRA; MASSAGUER(1999) \\
\hline Fortaleza, CE & Queijo de coalho & 84 & $16(19,0)$ & BRANCO et al. (2003) \\
\hline Campinas, SP & Minas frescal & 25 & $0(0,0)$ & Rocha (2004) \\
\hline Pernambuco ${ }^{Z}$ & Queijo de coalho & 127 & $7(5,5)$ & DUARTE et al. (2005) \\
\hline Fortaleza, CE & Queijo de coalho & 70 & $2(2,9)$ & Souza et al. (2006) \\
\hline Campinas, SP & Ricota & 45 & $3(6,7)$ & ESPER (2006) \\
\hline Rio Grande do Sul ${ }^{\mathrm{Z}}$ & Diversos & 80 & $3(3,8)$ & ZAFFARI et al. (2007) \\
\hline \multirow[t]{3}{*}{ Campinas, SP } & Minas frescal ${ }^{C L}$ & 31 & $3(9,7)$ & CARvalho et al. (2007) \\
\hline & Minas frescal ${ }^{\mathrm{AD}}$ & 31 & $0(0,0)$ & \\
\hline & Minas frescal ${ }^{\mathrm{UF}}$ & 31 & $0(0,0)$ & \\
\hline Serro, MG & Minas frescal do Serro & 40 & $0(0,0)$ & BRANT et al. (2007) \\
\hline Juiz de Fora, MG & Minas Frescal & 55 & $6(11,0)$ & BRITO et al. (2008) \\
\hline Paranáz & Diversos & 90 & $6(6,7)$ & ABRAÃo et al. (2008) \\
\hline Minas Gerais & Serra da Canastra & 27 & $0(0,0)$ & ANDRADE (2009) \\
\hline
\end{tabular}

$\mathrm{N}^{*}=$ número de amostras analisadas.

$\mathrm{n}(\%)=$ número e respectiva porcentagem de amostras positivas para L. monocytogenes.

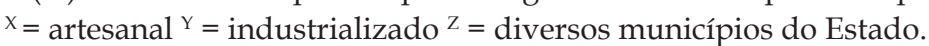

$\mathrm{CL}=$ cultura lática $\mathrm{AD}=$ acidificação direta $\mathrm{UF}=$ ultra-filtração 
Essa variação de ocorrência da bactéria em queijos (Tabela 2) pode ser explicada pelo uso de diferentes métodos para a detecção da bactéria, diferenças de padrões de qualidade dos queijos e pelo uso de leite cru ou pasteurizado como matéria-prima. Os coliformes parecem exercer influência na população de L. monocytogenes, em queijo Minas frescal, impedindo sua proliferação, podendo inclusive dificultar sua deteç̧ão (ArAgon-Alegro et al., 2006). A população baixa de L. monocytogenes e a injúria subletal causada pelo processamento são fatores que dificultam sua recuperação no caldo de enriquecimento. Assim, um resultado negativo não garante a ausência do patógeno, demonstrando a importância do plano de amostragem.

L. monocytogenes tem sido isolada de diversos tipos tradicionais de queijos em vários países, em frequências variáveis (PINTADOetal., 2005). A bactéria foi isolada em $6,4 \%$ (21/329) das amostras de queijos macios oriundas de países da Europa e, importante ressaltar, foi verificada maior ocorrência nos queijos elaborados com leite pasteurizado do que nos queijos fabricados com leite cru (RUDOLF; SCHERER, 2001), indicando que a contaminação ocorre durante o processamento na indústria.

Além de queijos, no Brasil há relato de isolamento de L. monocytogenes em uma amostra de creme de leite pasteurizado $(\mathrm{N}=19)$ (MARTINs et al., 2009), não tendo sido isolada de sorvetes (ABRAHÃo et al., 2008; MARTins et al., 2009).

\section{Listeria monocytogenes em indústrias de laticínios}

Os alimentos envolvidos em surtos de listeriose são, na maioria, industrializados. De fato, segundo FENLON et al. (1996), a fonte primária de L. monocytogenes nos alimentos industrializados é o ambiente e, através da matéria-prima vegetal ou animal, a bactéria é introduzida no ambiente industrial, onde certas cepas tornar-se-iam adaptadas. A partir de números não detectáveis, ela proliferaria durante o processamento. A cadeia produtiva do leite favorece a contaminação dos laticínios por L. monocytogenes. O leite cru pode ser uma fonte de introdução de $L$. monocytogenes na indústria (WAAK et al., 2002), mas há outras possibilidades como solas de calçados, vestuário e, possivelmente, portadores humanos saudáveis (ROCOURT; COSSART, 1997). As instalações e os equipamentos das indústrias de alimentos constituem fonte de contaminação, sobretudo quando não são adotadas Boas Práticas deFabricação(BPF), o que favorece o aparecimento de nichos de contaminação (TOMPKIN, 2002).

L. monocytogenes foi isolada de fábricas de queijos nos EUA que recebiam e trabalhavam exclusivamente com leite pasteurizado (KABUKI et al., 2004). Isso mostra a dificuldade do controle da
L. monocytogenes em laticínios, pois o controle de patógenos tradicionalmente é centrado na higiene de ordenha e pasteurização do leite mas, para esse micro-organismo, somente essas medidas não são, necessariamente, suficientes na sua prevenção, pois, se o laticínio não aplicar as BPF, recontaminações poderão ocorrer, especialmente em produtos muito manipulados como o queijo. WALKER et al. (1991) isolaram L. monocytogenes com maior frequência em laticínios que processavam grandes volumes deleite do que naqueles de pequeno porte, que trabalhavam com menores volumes, provavelmente devido ao tráfego intenso de pessoas, maiores dificuldades de controle e oportunidades de recontaminação.

No Brasil, L. monocytogenes tem sido isolada de ambiente de laticínios processadores de leite fluido (Figueiredo, 2000) e de queijos (Silva et al., 2003; BRITO et al., 2008; BARANCELLI et al., 2009) embora haja relatos de não isolamento da bactéria em ambientes de laticínio no país (RochA, 2004; Borges, 2006).

Há um interesse crescente na detecção de $L$. monocytogenes, sobretudo em indústrias dealimentos, devido à associação da bactéria presente no ambiente de processamento com o produto final. Estudos baseados em DNA têm mostrado que certas cepas de L. monocytogenes se estabelecem em indústrias de alimentos, onde permanecem residentes por meses ou anos, constituindo fontes permanentes de contaminação (MieTtinen et al., 1999; TOMPKIN, 2002). A persistência de cepas no ambiente industrial está relacionada com a adaptabilidade da bactéria e à provável formação de biofilmes (BAGGE-RAVN et al., 2003). Entretanto, não se sabe se essa persistência é resultado da adaptação de certos subtipos da bactéria, de limpeza e sanitização deficientes ou da habilidade do micro-organismo desenvolver tolerância a produtos utilizados no processo de higienização industrial (GRAM et al., 2007).

As grandes indústrias dealimentos vêm tentando minimizar o risco de veiculação de L. monocytogenes em produtos prontos para o consumo, porém esse risco ainda existe e não se sabe como as pequenas e microempresas estão tratando o problema (DESTRO, 2006). Em particular, para produtos de alto risco para L. monocytogenes, é importante que as indústrias adotem programa de coleta de amostras do ambiente (ILSI, 2005) e, a cada amostra positiva, seja para o gênero Listeria ou L. monocytogenes, as correções devem ser rápidas e efetivas, evitando assim a contaminação do alimento (TOMPKIN, 2002). A amostragem de produtos pode ser recomendada, durante o processamento, como parte do programa de vigilância, especialmente quando análises do ambiente resultarem positivas (ILSI, 2005).

Atualmente, pesquisas estão focadas no desenvolvimento de medidas de controle da bactéria em indústrias processadoras de alimentos (NATIONAL..., 
2009). Uma vez que o ingresso de L. monocytogenes na indústria pode ser contínuo, seu controle deve ser feitono seu interior com a aplicação de procedimentos de higienização e BPF (ToMPKIN, 2002). Indústrias que processam alimentos de alto risco de contaminação por L. monocytogenes devem operar com programas de Análise de Perigos e Pontos Críticos de Controle (APPCC) e Procedimentos Padronizados de Higiene Operacional (PPHO) para reduzir a contaminação ambiental e minimizar a contaminação dos produtos, prevenindo assim casos de listeriose humana (ILSI, 2005).

Outros pontos que merecem atençãona prevenção da listeriose são a comercialização dos alimentos no varejo e o ambiente doméstico, onde as práticas de higiene são fundamentais para evitar a contaminação durante a manipulação e o armazenamento. São necessárias campanhas educativas para manipuladores e consumidores de alimentos dos ambientes comerciais e residenciais, através da divulgação de informações sobre os riscos associados ao consumo de certos alimentos, como leite cru e queijos macios, especialmente para pessoas do grupo de risco. No caso de queijos frescos, isso é particularmente importante, uma vez que a população, de modo geral, considera esse produto "saudável" efrequentemente ele é indicado em dietas e consumido por crianças, idosos e convalescentes, sendo recomendado, muitas vezes, por profissionais da área de saúde.

\section{Necessidades e perspectivas}

Em alimentos prontos para o consumo, nos quais L. monocytogenes écapaz de se multiplicar, a principal tarefa é prevenir a contaminação. $\mathrm{O}$ uso de aditivos queinibem a bactéria representa uma garantia adicional para esses alimentos (TOMPKIN, 2002). Isso não é possível no leite pasteurizado, podendo ser aplicado para queijos frescos. O controle de L. monocytogenes na indústria é dificultado pelo fato da bactéria ser de origem ambiental, não havendo correlação entre a presença da bactéria e de micro-organismos indicadores higiênico-sanitários, como coliformes e Escherichia coli. Esse é um problema particularmente grave para os laticínios, já que usualmente são esses os indicadores utilizados no controle de qualidade microbiológica de ordenha, da eficiência da pasteurização e da sanidade de derivados, como queijos frescos. Assim, há necessidade da detecção de L. monocytogenes em produtos e amostras de ambiente para o controle efetivo do patógeno nas indústrias de laticínios.

Com relação à detecção de L. monocytogenes em alimentos, métodos baseados na reação em cadeia da polimerase (PCR) são uma alternativa para a limitação que os métodos convencionais de cultivo apresentam na detecção de baixos números. Com
PCR, a obtenção de resultado negativo, que é considerado definitivo, é mais rápida. Porém, quando o resultado é positivo, há necessidade de submeter a amostra ao isolamento e identificação do microorganismo, por métodos convencionais. A técnica de PCR pode ser mais sensível do que métodos convencionais e, assim, oferecer maior segurança ao consumidor (DESTRO, 2006). Por outro lado, pode produzir resultados falso-positivos, em decorrência da amplificação do DNA presente de células mortas.

Técnicas de subtipagem de L. monocytogenes baseadas em DNA são úteis para identificar alimentos incriminados em casos de listeriose e detectar fontes de contaminação, o que é necessário para elaboração e melhorias de medidas de controle da bactéria na cadeia de produção de alimentos. Técnicas de subtipagem possibilitam a definição de subtipos da bactéria, que diferem em suas características fenotípicas e/ou habilidade de causar doenças. Consequentemente, no futuro, somente certos subtipos de L. monocytogenes poderão ser considerados perigosos, quando presentes em alimentos prontos para o consumo (WIEDMANN 2002).

Outras necessidades são o aprimoramento dos métodos analíticos e estudos de predição do comportamento da bactéria, em alimentos e no ambiente industrial; melhorias no conhecimento sobre a contribuição dos diversos seguimentos da cadeia, da fazenda ao consumidor, na contaminação de alimentos, considerando a possível presença de animais decompanhia ehumanos como reservatórios assintomáticos, em residências; criação de bancos de dados de tipagem de L. monocytogenes, de fácil acesso para microbiologistas de agências governamentais, de instituições de pesquisa e da indústria, para fins de consultas e contribuição com dados. Espera-se que os estudos de genômica e proteômica possam permitir análises de isolados, com respeito a marcadores genéticos de cepas virulentas e expressão de proteínas, dos diversos sorotipos, bem como das características de linhagens especificamente preocupantes, como aquelas implicadas em surtos de listeriose (KATHARIOU, 2002).

A resistência de cepas de L. monocytogenes a antibióticos podecomprometer as opções de tratamentos. Segundo CONTER et al. (2009), existe pouca informação sobre a susceptibilidade a antimicrobianos de cepas deL.monocytogenes isoladas dealimentos eambientes de produção. Esses pesquisadores demonstraram que cepas oriundas de alimentos e ambientes de produção foram susceptíveis aos antibióticos comumenteusados em tratamentos das áreas médica e veterinária, mas alertam para a necessidade de vigilância contínua de resistência desse patógeno, para garantir eficiência nos tratamentos da listeriose humana.

É evidente que a ocorrência de listeriose no homem depende de propriedades inerentes ao agente 
como a dose infectante e a virulência da linhagem e, acima de tudo, de uma população consumidora de alimentos prontos para consumo cada vez mais portadora de fatores de risco predisponentes. As indústrias delaticínios, os profissionais de saúdeeos consumidores precisam ser alertados sobre os riscos da listeriose e suas respectivas responsabilidades no controle da L. monocytogenes em alimentos.

\section{REFERÊNCIAS}

ABRAHÃO, W.M.; ABRAHÃO, P.R. da S.; MONTEIRO, C.L.B.; PONTAROLO, R. Occurrence of Listeria monocytogenes in cheese and ice cream produced in the State of Paraná, Brazil. Revista Brasileira de Ciências Farmacêuticas, v.44, n.2, p.289-296, 2008.

ANDRADE, C.R. de Diagnóstico da qualidade microbiológica de queijo Serra da Canastra e caracterização de bactérias do gênero Enterococcus. 2009. 98f. Dissertação (Mestrado) - Universidade Estadual de Campinas, Campinas, 2009.

ARAGON-ALEGRO, L.C.; SOUZA, K.L.O.; NUNES T.P.; LANDGRAF, M.; FRANCO, B.D.G.M.; DESTRO, M.T. Influência de coliformes na população de Listeria monocytogenes em queijo Minas frescal. In: CONGRESSO BRASILEIRO DE CIÊNCIA E TECNOLOGIA DE ALIMENTOS, 10., 2006, Curitiba. Anais. Campinas: SBCTA, 2006.

ARCURI, E.F.; BRITO, M.A.V.P.; BRITO, J.R.F.; PINTO, S.M.; ANGELO, F.F.; SOUZA, G.N. Qualidade microbiológica do leite refrigerado nas fazendas. Arquivos da Sociedade Brasileira de Medicina Veterinária e Zootecnia, v.58, n.3, p.440-446, 2006.

AURELI, P.; FIORUCCI, G.C.; CAROLI, D.; MARCHIARO, G.; NOVARA, O.; LEONE, L.; SALMASO, S. An outbreak of febrile gastroenteritis associated with corn contaminated by Listeria monocytogenes. New England Journal of Medicine, v.342, n.17, p.1236-1241, 2000.

BAGGE-RAVN, D.; GARKSHODN, K.; GRAM, L.; VOGEL, B.F. Comparison of sodium hypochloritebased foam and peroxyacetic acid-base for sanitizing procedures in a salmon smokehouse: survival of general microflora and Listeria monocytogenes. Journal of Food Protection, v.66, n.4, p.592-598, 2003.

BARANCELLI, G.V.; CAMARGO, T.M.; ARRUDA, N.F.; BARBOSA, A.V.; PORTO, E.; OLIVEIRA, C.A.F.; HOFER, E. Ocorrência de Listeria monocytogenes em linha de produção e ambiente de processamento de queijo Minas frescal. In: CONGRESSO BRASILEIRO DE MICROBIOLOGIA, 25., 2009, Ipojuca. Anais. Ipojuca: SBM,2009. Resumo 1048. Disponível em: <http:// sbmicrobiologia.org.br/PDF/cdsbm/trabalhos.htm.> Acesso em: 5 dez. 2009.

BEMRAH, N.; SANNA, M.; CASSIN, M.H.; GRIFFITHS, M.W.; CERF, O. Quantitative risk assessment of human listeriosis from consumption of soft cheese made from raw milk. Preventive Veterinary Medicine, v.37, n.1/4, p.129-145, 1999.

BILLE, J.; BLANC, D.S.; SHIMID, H.; BOUBAKER, K.; BAUMGARTNER, A.; SIEGRIST, H.H.; TRITTEN, M.L.; LIENHARD, R.; BERNER, D.; ANDERAU, R.; TREBOUX, M.; DUCOMMUN, J.M.; MALINVERNI, R.; GENNÉ, D.; ERARD, P.; WAESPI, U. Outbreak of human listeriosis associated with tomme cheese in northwest Switzerland, 2005. Eurosurveillance, v.11, n.6, p.91-93, 2006. Disponível em: <http:/ / www. eurosurveillance.org/ViewArticle.aspx?ArticleId=633>. Acesso em 5 dez. 2009.

BORGES, MF. Diagnóstico da contaminação por bactérias patogênicas em indústria de processamento de queijo de coalho e detecção de genes associados a fatores de virulência. Campinas, 2006. 199f. Tese (Doutorado) - Faculdade de Engenharia de Alimentos, Universidade Estadual de Campinas, Campinas, 2006.

BRANCO, M.A.A.C.; FIGUEIREDO, A.T.; BORGES, M.F. SILVA, M.D.D.; DESTRO, M.T. Incidência de Listeria monocytogenes em queijo de coalho refrigerado produzido artificialmente. B CEPPA, v.21, n.2, p.393-408, 2003.

BRANT, L.M.F.; FONSECA, L.M.; SILVA, M.C.C. Avaliação da qualidade microbiológica do queijo-de-minas artesanal do Serro-MG. Arquivo Brasileiro de Medicina Veterinária e Zootecnia, v.59, n.6, p.1570-1574, 2007.

BRASIL. Ministério da Agricultura, do Abastecimento e da Reforma Agrária. Portaria n 146, de 07 de março de 1996. Aprova regulamentos técnicos de indentidade e qualidade dos produtos lácteos. Diário Oficial da União, Brasília, 11 mar. 96. Seção I. 50p.

BRITO, J.R.; SANTOS, E.M.P.; ARCURI, E.F.; LANGE, C.C.; BRITO, M.A.V.P. SOUZA, G.N.; CERQUEIRA, M.M.P.O. MARCELA SOTO BELTRAN, J.; CALL, J.E.; LIU, Y.; PROTO-FEET, A.C.S.; LUCHANSKY, J.B. Retail survey of brazilian milk and minas frescal cheese and a contaminated dairy plant to establish prevalence, relatedness, and sources of Listeria monocytogenes isolates. Applied and Environmental Microbiology, v.74, n.15, p.4954-4961, 2008.

CAMARGO, T.M. Prevalência de Listeria monocytogenes, coliformes totais e Escherichia coli em leite cru refrigerado e ambiente de ordenha de propriedades leiteiras do Estado de São Paulo. Piracicaba, 2010. 104f. Dissertação (Mestrado) - Escola Superior de Agricultura Luiz de Queiroz, Universidade de São Paulo, Piracicaba, 2010.

CARRIQUE-MAS J.J.; HÖKEBERG, I.; ANDERSON, Y.; ARNEBORN, M.; THAM, W.; DANIELSSON-THAM, M.L.; OSTERMAN, B.; LEFFLER, M.; STEEN, N.; ERIKSSON, E.; HEDIN, G.; GIESECKE, J. Febrile gastroenteritis after eating on-farm manufactured fresh cheese - an outbreak of listeriosis? Epidemiology and Infection, v.130, n.1, p.79-86, 2003. 
CARVALHO, J.D.G.; VIOTTO, W.H.; KUAYNE, A.Y. The quality of Minas Frescal cheese produced by different technological processes. Food Control, v.18, n.2, p.262-267, 2007.

CASAROTTI, V.T.; GALLO, C.R.; CAMARGO, R. Ocorrência de Listeria monocytogenes em leite cru, leite pasteurizado tipo C e queijo minas frescal comercializados em Piracicaba-SP. Archivos Latinoamericanos de Nutrición, v.44, n.3, p.158-163, 1994.

CATÃO, R.M.R.; CEBALLOS, B.S.O. Listeria spp., coliformes totais e fecais e E. coli no leite cru e pasteurizado de uma indústria de laticínios, no estado da Paraíba (Brasil). Ciência e Tecnologia de Alimentos, v.21, n.3, p.281-287, 2001.

CENTER FOR DISEASE CONTROL AND PREVENTION. Preliminary FoodNet Data on the Incidence of Infection with Pathogens Transmitted Commonly Through Food 10 States, 2007. 2007. Disponível em: <http:/ / www.cdc. gov/mmwr/preview/mmwrhtml/mm5714a2.htm>.

Acesso em: 3 nov. 2010.

CENTER FOR DISEASE CONTROL AND PREVENTION. Outbreak of Listeria monocytogenes Infections Associated with Pasteurized Milk from a Local Dairy - Massachusetts, 2007. 2008. Disponível em: <http://www.cdc.gov/ $\mathrm{mmwr} / \mathrm{preview} / \mathrm{mmw} \mathrm{rhtml} / \mathrm{mm} 5740 \mathrm{a} 1 . \mathrm{htm}>$. Acesso em: 10 mar. 2009.

CENTRO DE VIGILÂNCIA EPIDEMIOLÓGICA. São Paulo. Informações sobre doenças transmitidas por água e alimentos. Listeria monocytogenes/listeriose. 2003. Disponível em: <http:/ / www.cve.saude.sp.gov.br/ htm/hidrica/Listeria.htm>. Acesso em: 8 mai. 2008.

CHAMBEL, L.; SOL, M.; FERNANDES, I.; BARBOSA, M.; ZILHÃO, i.; BARATA, B.; JORDAN, S.; PERNI, S.; SHAMA, G.; ADRIÃO, A.; FALEIRO, L.; REQUENA, T.; PELÁEZ, C.; ANDREW, P.W.; TENREIRO, R. Occurrence and persistence of Listeria spp. in the environment of ewe and cow's milk cheese dairies in Portugal unveiled by an integrated analysis of identification, typing and spatialtemporal mapping along production cycle. International Journal of Food Microbiology, v.116, n.1, p.52-63, 2007.

CONTER, M.; PALUDI, D.; ZANARDI, E.; GHIDINI, S.; VERGARA, A.; IANIERI, A. Characterization of antimicrobial resistance of foodborne Listeria monocytogenes. International Journal of Food Microbiology, v.128, n.3, p.497-500, 2009.

DALTON C.B. AUSTIN, C.C.; SOBEL, J.; HAYES, P.S.; BIBB, W.F.; GRAVES, L.M.; SWAMINATHAN, B.; PROCTOR, M.E.; GRIFFIN, P.M. An outbreak of gastroenteritis and fever due to Listeria monocytogenes in milk. New England Journal of Medicine, v.336, n.2, p.100-105, 1997.

DE SÁ, F.R.N.; SZTAJNBOK, J.; DE ALMEIDA, J.F.L.; TROSTER, E.J.; VAZ, F.A.C. Listeria monocytogenes pneumonia in a cirrhotic child. International Journal of Clinical Practice, v.58, n.5, p.536-538, 2004.
DESTRO, M.T. Listeria monocytogenes na cadeia produtiva de alimentos: da produção primária ao consumidor final. 2006. 74f. Tese (Livre Docência) - Faculdade de Ciências Farmacêuticas, Universidade de São Paulo, São Paulo, 2006.

DESTRO, M.T.; MELO SERRANO, A. de; KABUKI, D.Y. Isolation of Listeria species from some Brazilian meat and dairy products. Food Control, v.2, n.2, p.110-112, 1991.

DOMINGUEZ-RODRIGUEZ, L.; GARAYZABAL, J.F.F.; VAZQUEZ-BOLAND, J.A.; FERRI, E.R.;

FERNÁNDEZ, G.S. Isolation of microorganisms of the species Listeria from raw milk intended for human consumption. Canadian Journal of Microbiology, v.31, n.10, p.938-941, 1985.

DUARTE, D.A.M.; SCHUCH, D.M.T.; SANTOS, S.B.; RIBEIRO, A.R.; VASCONCELOS, A.M.M.; SILVA, J.V.D.; MOTA, R.A. Pesquisa de Listeria monocytogenes e microrganismos indicadores higiênico-sanitários em queijo de coalho produzido e comercializado no estado de Pernambuco. Arquivos do Instituto Biológico, São Paulo, v.72, n.3, p.297-302, 2005.

ESPER, L.M.R. Diagnóstico da qualidade de ricotas comercializadas no município de Campinas - SP. 2006. 97f. Dissertação (Mestrado em Tecnologia de Alimentos) Universidade Estadual de Campinas, Campinas, 2006.

ESPER, M.R.N.R., PESSOA, G.V.A.; HOFER, E.; LEE, I.M.L.; MELLES, C.E.A.; SAKATA, E.E.; CALZADA, C.T. Meningite por Listeria monocytogenes em São Paulo, Brasil. Revista do Instituto Adolfo Lutz, v.38, n.1, p.37-41, 1978.

FAO/WHO (Food and Agriculture Organization of the United Nations / World Health Organization) Risk assessments of Listeria monocytogenes in ready-to-eat foods: technical report. Geneva: FAO/WHO; 2004. 269p.

FARBER, J.M.; PETERKIN, P.I. Listeria monocytogenes, a food-borne pathogen. Microbiological Reviews, v.55, n.3, p.476-511, 1991.

FENLON, D.R.; WILSON, J.; DONACHIE, W. The incidence and level of Listeria monocytogenes contamination of food sources at primary production and initial processing. Journal of Applied Bacteriology, v.81, n.6, p.641-650, 1996.

FIGUEIREDO, E.A.T. Ocorrência do gênero Listeria e avaliação da diversidade genética de Listeria monocytogenes através do random amplified polymorphic DNA (RAPD) e sua distribuição em linha de processamento de leite pasteurizado tipo " $C$ ". 2000. 100f. Tese (Doutorado em Microbiologia) - Instituto de Ciências Farmacêuticas, Universidade de São Paulo, São Paulo, 2000.

FLEMMING, D.W. Pasteurized milk as a vehicle of infection in an outbreak of listeriosis. New England Journal of Medicine, v.312, n.7, p.404-407, 1985. 
FOOD SAFETY AUTHORITY OF IRELAND The control and management of Listeria monocytogenes contamination of food. Dublin: FSAI, 2005. 94p. (Technical Report) Disponível em: <http://www.fsai.ie/publications/reports/ listeria_report.pdf $>$. Acesso em: 10 mar. 2009.

FRETZ, R.; SAGEL, U.; RUPPITSCH, W.; PIETZKA, A.T.; STÖGER, A.; HUHULESCU, S.; HEUBERGER, S.; PICHLER, J.; MUCH, P.; PFAFF, G.; STARK, K.; PRAGER, K.; FLIEGER, A.; FEENSTRA, O.; ALLERBERGER, F. Listeriosis outbreak caused by acid curd cheese 'Quargel' Austria and Germany 2009. Eurosurveillance, v.15, n.5, p.1-2, 2010. Disponível em: <http:/ / www.eurosurveillance.org/ViewArticle.aspx?ArticleId=19477>. Acesso em 25 out. 2010.

FURLANETTO, S.M.P.; SANTOS, M.A.A.; HARA, C. Listeria spp: Avaliação da eficiência de quatro meios de plaqueamento no seu isolamento. Higiene Alimentar, v.10, n.46, p.30-34, 1996.

GAULIN, C. First documented outbreak of Listeria monocytogenes in Quebec, 2002. Canadá. Communicable Disease Report. 2003; v.29, n.21, 2003. Disponível em: <http://www.phac-aspc.gc.ca/publicat/ccdrrmtc/03vol29/dr2921ea.html>. Acesso em: 20 ago. 2007.

GRAM, L.; BAGGE-RAUN, D.; NG, Y.Y.; GYMOESE, P.; VOGEL, B.F. Influence of food soiling matrix on cleaning and disinfection efficiency on surface attached Listeria monocytogenes. Food Control, v.18, n.10, p.11651171, 2007.

GOLNAZARIAN, C.; DONNELY, C.W.; PITAURO, S.J.; HOWARD, D.B. Comparison of infectious dose of Listeria monocyotenes $\mathrm{F} 5817$ as determined for normal versus compromised C67B1/6J mice. Journal of Food Protection, v.52, n.10, p.696-701, 1989.

GOULET, V.; JACQUET, C.; VAILLANT, V.; REBIÈRE, I.; MOURET, E.; LORENTE, C.; MAILLOT, E.; STAİNER, F.; ROCOURT, J. Listeriosis from consumption of raw-milk cheese. The Lancet, v.345, n.8964, p.1581$1582,1995$.

HEISICK, J.E.; ROSAS-MARTY, L.; TATINI, S.R. Enumeration of viable Listeria species and Listeria monocytogenes in foods. Journal of Food Protection, v.58, n.7, p.733-736, 1995.

HOF, H. Therapeutic options. FEMS Immunology and Medical Microbiology, v.35, n.3, p.203-205, 2003a.

HOF, H. History and epidemiology of listeriosis. FEMS Immunology and Medical Microbiology, v.35, n.3, p.199202, 2003b.

HOFER, E, MENEZES, D.M.F. de Isolamento de Listeria monocytogenes em secreção vaginal. In: CONGRESSO BRASILEIRO DE MICROBIOLOGIA, 1., 1969, Rio de Janeiro. Resumos. Rio de Janeiro: SBM, 1969. p.158.
HOFER, E.; PESSÔA, G.V.A.; MELLES, C.E.A. Listeriose humana. Prevalência de sorotipos de Listeria monocytogenes isolados no Brasil. Revista do Instituto Adolfo Lutz, v.44, n.2, p.125-131, 1984.

HOFER, E.; NASCIMENTO, R.S.; OLIVEIRA, M.A. Meningite por Listeria monocytogenes. Relato de casos em pacientes do Distrito Federal. Revista da Sociedade Brasileira de Medicina Tropical, v.31, n.2, p.173-177, 1998.

HOFER, C.B.; MELLE, C.E.A.; HOFER, E. Listeria monocytogenes in renal transplant recipients. Revista do Instituto de Medicina Tropical, v.41, n.6, p.375-377, 1999.

HOFER, E.; RIBEIRO, R.; FEITOSA, D.P. Species and serovars of the genus Listeria isolated from different sources in Brazil from 1971 to 1997. Memórias do Instituto Oswaldo Cruz, v.95, n.5, p.615-620, 2000.

HOFER, E.; REIS, C.M.F.; HOFER, C.B. Sorovares de Listeria monocytogenes e espécies relacionadas isoladas de material clínico humano. Revista da Sociedade Brasileira de Medicina Tropical, v.39, n.1, p.32-37, 2006.

ILSI. Research foundation/risk science institute, expert panel on Listeria monocytogenes in foods. Achieving continous improvement in reduction in foodborne listeriosis - a risk based approach. Journal of Food Protection, v.68, n.9, p.1932-1994, 2005.

IVANEK, R.; GRÖHN, Y.T.; WIEDMANN, M. Listeria monocytogenes in multiple habitats and host populations: review of available data for mathematical modeling. Foodborne Pathogens and Disease, v.3, n.4, p.319-336, 2006.

JAY, J.M. Modern food microbiology. 6.ed. Gaithersburg: Aspen Publishers, 2000. 679p.

JACQUET, C.; CATIMEL, B.; BROSCH, R.; BUCHRIESER, C.; DEHAUMONT, P.; GOULET, V.; LEPOUTRE, A.; VEIT, P.; ROCOURT, J. Investigations related to the epidemic strain involved in the French listeriosis outbreak in 1992. Applied and Environmental Microbiology, v.61, n.6, p.2242-2246, 1995.

JEFFERS, G.T.; BRUCE, J.L.; McDONOUGH, J.S.; BOOR, K.J.; WIEDMANN, M. Comparative genetic characterization of Listeria monocytogenes isolates from human and animal listeriosis case. Microbiology, v.147, n.5, p.1095-1104, 2001.

KABUKI, D.Y.; KUAYE, A.Y.; WIEDMANN, M.; BOOR, K.J. Molecular subtyping and tracking of Listeria monocytogenes in latin-style fresh-cheese processing plants. Journal of Dairy Science, v.87, n.9, p.2803-2812, 2004.

KATHARIOU, S. Listeria monocytogenes virulence and pathogenicity, a food safety perspective. Journal of Food Protection, v.65, n.11, p.1811-1829, 2002. 
KOZAK, J.; BALMER, R.; BYRNE, R.; FISHER, K. Prevalence of Listeria monocytogenes in foods: incidence in dairy products. Food Control, v.7, n.4/5, p.215-221, 1996.

LAKE, R.; HUDSON, A.; CRESSEY, P.; GILBERT, S. Risk Profile: Listeria monocytogenes in processed ready-to-eat salads/Institute of Environmental Science \& Research Limited. New Zeland. 2005. 68p. (Technical Report) Disponível em: <http:/ / www.nzfsa.govt.nz/ science/risk-profiles/FW0446_L_mono_in_RTE_salads_2005.pdf>. Acesso em: 1 abr. 2008.

LANDGRAF, I.M.; KOBATA, A.M.M.; JAKABI, M.; KIRSCHBAUM, C.R.A.; MARCHI, C.R. Surto de meningite neonatal por Listeria monocytogenes. Revista do Instituto Adolfo Lutz, v.58, n.1, p.63-67, 1999.

LEMES-MARQUES, E.G.; CRUZ, C.D.; DESTRO, M.T. Pheno and genotypic characterization of Listeria monocytogenes clinical isolates from the southwestern region of the State of São Paulo, Brazil. Brazilian Journal of Microbiology, v.38, n.2, p.287-292, 2007.

LIANOU, A.; SOFOS, J.N. A review of the incidence and transmission of Listeria monocytogenes in ready-toeat products in retais and food service environments. Journal of Food Protection, v.70, n.9, p.2172-2198, 2007.

LINNAN, M.J.; MASCOLA, L.; LOU, X.D.; GOULET, V.; MAY, S.; SALMINEN, C.; HIRD, D.W.; YONEKURA, L.; HAYES, P.; WEAVER, R.; AUDURIER, A.; PLIKAYTIS, B.D.; FANNIN, S.L.; KLEKS, A.; BROOME, C.V. Epidemic listeriosis associated with Mexican-style cheese. New England Journal of Medicine, v.319, n.13, p.823-828, 1988.

LYYTIKÄINEN, O.; AUTIO, T.; MAIJALA, R.; RUUTU, P.; HOKANEN-BUZALSKI, T.H.; MIETTINE, M.; HATAKKA, M.; MIKKOLA, J.; ANTILA, V.; JOHANSSON, T.; RANTALA, 1.; AALTO, T.; KORKEALA, H.; SIITONEN, A. An outbreak of Listeria monocytogenes serotype 3a infections from butter in Finlandia. Journal of Infectious Diseases, v.181, n.5, p.1838-1841, 2000.

MAKINO, S.I.; KAWAMOTO, K.; TAKESHI, K.; OKADA, Y.; YAMASAKI, M.; YAMAMOTO, S. An outbreak of food-borne listeriosis due to cheese in Japan, during 2001. International Journal of Food Microbiology, v.104, n.2, p.189-196, 2005.

MARTINS, I.M.; KABUKI, D.Y.; KUAYE, A.Y. Determination and characterization of pathogens found in dairy products. Revista do Instituto Adolfo Lutz, v.68, n.3, p.359-365, 2009.

MARTH, E.H. Disease characteristics of Listeria monocytogenes. Food Technology, v.42, n.4, p.165-169, 1988.

MASSA, S.; CESARONI, D.; PODA, G.; TROVATELLI, L.D. The incidence of Listeria spp. in soft cheeses, butter and raw milk in the province of Bologna. Journal of Applied Bacteriology, v.68, n.2, p.153-156, 1990.
McDONALD, P.D.M.; WHITWAM, R.E.; BOGGS, J.D.; MacCORMACK, J.N.; ANDERSON, K.L. REARDON J.W.; SAAH, J.R. GRAVES, L.M.; HUNTER, S.B.; SOBEL, J. Outbreak of listeriosis among Mexican immigrants as a result of consumption of illicitly produced Mexican-style cheese. Clinical Infectious Disease, v.40, n.5, p.677-682, 2005.

McLAUCHLIN J. The relationship between Listeria and listeriosis. Food Control, v.7, n.4/5, p.187-193, 1996.

MEAD, P.S.; SLUTSKER, L.; DIETZ, V.; M; McCAIG, L.F.; BRESSEL, J.S.; SHAPIRO, C.; GRIFFIN, P.M.; TAUXE, R.V. Food-related illness and death in the United States. Emerging Infectious Diseases, v.5, n.5, p.607-625, 1999.

MEYER-BROSETA, S.; DIOT, A.; BASTIAN, S.; RIVIÈRE, J.; CERF, O. Estimation of low bacterial concentration: Listeria monocytogenes in raw milk. International Journal of Food Microbiology, v.80, n.1, p.1-15, 2003.

MIETTINEN, M.K.; BJÖRKROTH, K.J.; KORKEALA, H.J. Characterization of L. monocytogenes from an ice cream plant by serotyping and pulsed-field gel electrophoresis. International Journal of Food Microbiology, v.46, n.3, p.187-192, 1999.

MOURA, S.M.; DESTRO, M.T.; FRANCO, B.D.G.M. Incidence of Listeria species in raw and pasteurized milk produced in São Paulo, Brasil. International Journal of Food Microbiology, v.9, n.3, p.229-237, 1993.

MURAOKA, W.; GAY, C.; KNOWLES, D.; BORUCKI, M. Prevalence of Listeria monocytogenes subtypes in bulk milk of the pacific northwest. Journal of Food Protection, v.66, n.8, p.1413-1419, 2003.

NACIONAL AGRICULTURAL LIBRARY (US). National Agricultural Library. A Focus on Listeria monocytogenes. 2009. Disponível em: <http://fsrio.nal. usda.gov/document_fsheet.php?product_id=221>. Acesso em: 30 nov. 2009.

NERO, L.A. Listeria monocytogenes e Salmonella spp. em leite cru produzido em quatro regiões leiteiras no Brasil: ocorrência e fatores que interferem na sua detecção. 2005. 141f. Tese (Doutorado em Ciências dos Alimentos) - Faculdade de Ciências Farmacêuticas, Universidade de São Paulo, São Paulo, 2005.

PINTADO, C.M.B.S.; OLIVEIRA, A.; PAMPULHA, M.E.; FERREIRA, M.A.S.S. Prevalence and characterization of Listeria monocytogenes isolated from soft cheese. Food Microbiology, v.22, n.1, p.79-85, 2005.

RADOSTITS, O.M.; BLOOD, D.C.; GAY, C.C. Veterinary medicine. London: Baillière Tindall, 1994. 1763p.

ROCHA, J.A.K. Estudo da presença de Listeria monocytogenes e Bacillus cereus em indústria processadora de queijo minas frescal. 2004. 77f. Dissertação (Mestrado) - Faculdade 
de Engenharia de Alimentos, Universidade Estadual de Campinas, Campinas, 2004.

ROCOURT, J.; COSSART, O. Listeria monocytogenes In: DOYLE, M.P.; BEUCHAT, L.R.; MONTVILLE, T.J. Ed). Food Microbiology: fundamentals and frontiers. Washington: ASM Press, 1997. p.337-352.

ROCOURT, J.; BEN EMBAREK, P.; TOYOUFUKU, H.; SCHLUNDT, J. Quantitative risk assessment of Listeria monocytogenes in ready-to-eat: the FAO/WHO approach. FEMS Immunology and Medical Microbiology, v.35, n. 3, p.263-267, 2003.

ROCOURT, J.; BUCHRIESER, C. The genus Listeria and Listeria monocytogenes: phylogenetic position, taxonomy, and identification. In: RYSER, E.T.; MARTH, E.H. (Ed.). 3.ed. Listeria, listeriosis and food safety. Boca Raton: CRC Press, 2007. Chap. 1, p.1-20.

RUDOLF, M.; SCHERER, S. High incidence of L. monocytogenes in European red smear cheese. International Journal of Food Microbiology, v.63, n.1/2, p.91-98, 2001.

RYSER, E.T.; MARTH, E.H. (Ed). Listeria, listeriosis, and food safety. New York: Marcel Dekker, 1991. 632p.

RYSER, E.T.; DONNELY, C.W. Listeria. In: DOWNES, F.P.; ITO, K.(Ed.). Compendium of methods for the microbiological examination on foods. 4.ed. Washington: American Public Heath Association, 2001. p.343-356. Chap. 36.

SCHLECH, W.F. Virulence characteristics of Listeria monocytogenes. Food Technology. v.42, n.4, p.176-178, 1988.

SCHWAB, J.P.; EDELWEISS, M.I.A. Identificação imunohistoquímica de Listeria monocytogenes em placentas fixadas em formol e embebidas em parafina. Revista Brasileira de Ginecologia e Obstetrícia, v.25, n.7, p.501-505, 2003.

SEELIGER, H.P.R. Listeriosis. New York: Hafner Publ., 1961. 308p.

SILVA, M.C.D.; HOFER, E.; TIBANA, A. Incidence of Listeria monocytogenes in cheese produced in Rio de Janeiro, Brazil. Journal of Food Protection, v.61, n.3, p.354356, 1998.

SILVA, I.M.M.; ALMEIDA, R.C.C.; ALVES, M.A.O.; ALMEIDA, P.F. Ocurrence of Listeria spp. in critical control points and the environment of minas frescal cheese processing. International Journal of Food Microbiology, v.81, n.3, p.241-248, 2003.

SILVA, N.; JUNQUEIRA, V.C.A.; SILVEIRA, N.F.A; TANIWAKI, M.H.; SANTOS, R.F.S.; GOMES, R.A.R. Manual de métodos de análise microbiológica de alimentos. 3.ed. São Paulo: Varela, 2007. 536p.

SLUTSKER, L.; SCHUCHAT, A. Listeriosis in humans. In: RYSER, E.T.; MARTH, E.H. Listeria, listeriosis and food safety. 2.ed. New York: Marcel Dekker, 1999. p.75-95.
SOUZA, R.A.; FIGUEIREDO, E.A.T.; MAIA, G.A.; FRIZ-ZO, S.E. Incidência de Listeria monocytogenes em queijo de coalho artesanal, comercializado à temperatura ambiente em Fortaleza, CE. Higiene Alimentar, v.20, p.66-69, 2006.

STEPHENS, J.C.; ROBERTS, I.S.; JONES, D.; ANDREW, P.W. Effect of growth temperature on virulence of strains of Listeria monocytogenes in the mouse: evidence for a dose dependence. Journal of Applied Microbiology, v.70, n.3, p.239-244, 1991.

SUASSUNA, I.; SANTOS, L.C.; SUASSUNA, I.R.; PINHEIRO, J. Listeriose do sistema nervoso no Estado da Guanabara. In: CONGRESSO BRASILEIRO DE MICROBIOLOGIA, 1., 1969, Rio de Janeiro. Resumos. Rio de Janeiro: SBM, 1969. p.161.

SWAMINATHAN, B.; GERNER-SMIDT, P. The epidemiology of human listeriosis. Microbes and Infection, v.9, n.10, p.1236-1243, 2007.

TRANT, T.; HITCHINS, A.D. Evaluation of a selective enrichment most probable number method for viable Listeria spp. in dairy products. Journal of Food Protection, v.59, n.9, p.928-931, 1996.

TOMA, B.; DUFOUR, B.; SANAA, M.; BENET, J.J.; MOUTOU, F.; LOUZA, A.; ELLIS, P. Applied veterinary epidemiology and the control of disease in populations. Maisons-Alfort, France: AEEMA, 1999. 536p.

TOMPKIN, R.B. Control of Listeria monocytogenes in the food-processing environment. Journal of Food Protection, v.65, n.4, p.709-725, 2002.

TOYOSHIMA, M.T.K. APANAVICIUS, A.; SOEIRO, A.M.A.; ALMEIDA, G.M.D. de, ARAI, M.H. Listeria monocytogenes peritonitis in cirrhotic patients: first description in Brazil. Revista do Instituto de Medicina Tropical, v.48, n.5, p.291-293, 2006.

UNITED STATES. Department of Health and Human Services. Quantitative assessment of the relative risk to public health from foodborne Listeria monocytogenes among selected categories of ready-to-eat foods. 2003 Disponível em: <http:/ /www.foodsafety.gov/;dms / lmr2-toc.html>. Acesso em: 20 fev. 2005.

UNITED STATES. Food and Drug Administration. Bad bug book: foodborne pathogenic microorganisms and natural toxins handbook. chapt.6.Listeria monocytogenes. 2007. Disponível em: <http:/ / www. cfsan.fda.gov/ mow/chap6.html>. Acesso em: 30 nov. 2009.

UNITED STATES. Food and Drug Administration. Quantitative assessment of relative risk to public health from foodborne Listeria monocytogenes among selected categories of ready-to-eat foods. 2003. Disponível em: <http://www.cfsan.fda.gov/ dms/lmr2-toc.html>. Acesso em: 10 nov. 2007. 
VAN KESSEL, J.S.; KARNS, J.S.; GORSK, L.; MCCLUSKEY, B.J.; PERDUE, M.L. Prevalence of Salmonellae, Listeria monocytogenes and fecal coliforms in bulk tank milk on US dairies. Journal of Dairy Science, v.87, n.9, p.2822-2830, 2004.

VIEIRA, M.A.S.; MASSAGUER, P.R. Incidência de Listeria ssp. em queijos minas frescal comercializados em Campinas/SP. Revista Indústria de Laticínios, v.23, n.4, p.62-65, 1999.

WAAK, E.; THAM, W.; DANIELSSON-THAM, M. Prevalece and Fingerprinting of Listeria monocytogenes strains isolated from raw whole milk in farm bulk tanks and in dairy plant receiving tanks. Applied and Environmental Microbiology, v.68, n.7, p.3366-3370, 2002.
WALKER, R.L.; JENSEN, L.; KINDE, H.; ALEXANDER, A.V.; OWENS, L. Environmental survey for Listeria species in frozen milk product plants in California. Journal of Food Protection, v.54, n.3, p.178-182, 1991.

WIEDMANN, M. Molecular subtyping methods for Listeria monocytogenes. Journal of Association of Official Analytical Chemists, v.85, n.2, p.524-531, 2002.

ZAFFARI, C.B.; MELLO, J.F.; COSTA, M. Qualidade bacteriológica de queijos artesanais comercializados em estradas do litoral norte do Rio Grande do Sul, Brasil. Ciência Rural, v.37, n.3, p.862-867, 2007.

Recebido em 18/12/09

Aceito em 5/11/10 